\title{
Seismic wavelet estimation via a system identification method*
}

\author{
Shaoshui Wang * Yongshou Dai and Fang Wang \\ College of Information and Control Engineering, China University of Petroleum, Dongying 257061, China
}

\begin{abstract}
On the assumption that the wavelet is causal and nonminimum phase, an autoregressive moving average (ARMA) model is introduced to fit the seismic trace. Seismic wavelet extraction is converted to parameters estimation of the ARMA model. Singular value decomposition (SVD) of an appropriate matrix formed by autocorrelation is exploited to determine the autoregressive (AR) order, and the cumulant-based SVD-TLS (total least squares) approach is proposed to obtain the AR parameters. The author proposes a new moving average (MA) model order determination method via combining the information theoretic criteria method and higher-order cumulant method. The cumulant approach is used to achieve the MA parameters. Theoretical analysis and numerical simulations demonstrate the feasibility of the wavelet extraction approach.
\end{abstract}

Key words: seismic wavelet; ARMA model; SVD; higher order cumulant; information theoretic criteria CLC number: P315. $3^{+} 1 \quad$ Document code: A

\section{Introduction}

Seismic wavelet estimation is the base of wave impedance inversion and forward modeling. Currently there are two broad categories of wavelet estimation, i.e., deterministic wavelet estimation and statistical wavelet estimation. In order to achieve a high resolution seismic wavelet, it is necessary to use the parametric statistical method, which is related to system identification.

During recent years, higher order cumulant based parameterized seismic wavelet estimation has been developed greatly. Lazear (1993) firstly introduced the cumulant based moving average (MA) model into seismic wavelet estimation. He obtained a mixed-phase, non-causal seismic wavelet by solving a cost function with the gradient decent method in a minimum mean-squared error sense. Following Lazear's work, relevant researchers applied better global optimization algorithms or order estimation methods to enhance the accuracy and stability of the estimation result (Velis and Ulrych, 1996). However, the computational prices of these methods are still high because they are all based on the MA model description. According to the Wold decomposition theorem (Zhang, 2002), Dai et al (2006) first introduced the autoregressive moving average (ARMA)

\footnotetext{
* Received 15 April 2009; accepted in revised form 1 September 2009; published 10 October 2009.

* Corresponding author. e-mail: wss.2008@163.com
}

model into modeling seismic wavelet. Compared with the MA model, the orders $p$ and $q$ of the ARMA model have no direct relationship with the real length of the seismic wavelet. We can achieve a precise seismic wavelet via fewer parameters. We call this feature of the ARMA model as that the parameter is parsimonious.

The actual seismic wavelet is non-causal, but the causal components are dominant and the non-causal components introduced by the detector and the channels are small and unknown. If we intervene to determine the extent of its non-causality, it may cause actual seismic wavelet to be distorted further. It is necessary to study the causal wavelet in detail, and then research the characteristics and applications of non-causal systems.

Following Dai's work (Dai et al, 2006), the author introduces the ARMA model to fit the seismic trace, and research on the order determination as a key role. In this paper singular value decomposition (SVD) is used to determine the rank of an appropriate matrix formed by autocorrelation as the AR order. The author proposes a new MA model order determination method via combining the information theoretic criteria method and higher- order cumulant method. After getting the correct order, the parameters of the AR and MA parts are estimated, respectively, by the SVD-TLS and the cumulant based approaches. The effectiveness of this approach is demonstrated using numerical simulations and real seismic data examples. 


\section{Seismic convolution model (MA model)}

It is generally considered that the seismic trace $y(n)$ is assumed to be a zero-mean stationary stochastic process, and is formulated as a convolution plus additive noise $v(n)$, often represented by the well-known convolutional model

$$
y(n)=\sum_{i=0}^{q} w(i) r(n-i)+v(n)=w(n) * r(n)+v(n),
$$

where $w(n)$ is mixed phase seismic wavelet, $q$ denotes the length of causal wavelet; the reflection coefficient series $r(n)$ is a stationary, zero-mean, independent and identically distributed, non-Gaussian process; and $v(n)$ is the additive noise, whose colored Gaussian components are dominant, independent of $r(n)$.

Dai et al (2006) employed the cumulant based matrix-equation approach to estimate the wavelet based on the MA model. Simulations show that the maximum mean square error of the model parameters increases rapidly when the length of the wavelet is larger than eight. However, the length of the real seismic wavelet is more than $70 \mathrm{~ms}$; when the sampling interval of seismic trace is $1 \mathrm{~ms}$, more than 70 parameters should be estimated for the MA model description.

According to the signal theory, we know that equation (1) is a typical MA model system, i.e., the finite impulse response (FIR) system. The number of the model parameters (i.e., $q$ ) is just the length of the wavelet, and the values of the model parameters are just the amplitude values of the wavelet.

\section{ARMA model definition}

Considering the effective (i.e., noise free) wave $x(t)$ as a stationary and ergodic linear process specified by

$$
\sum_{i=0}^{p} a(i) x(n-i)=\sum_{j=0}^{q} b(j) r(n-j),
$$

the effective wave $x(n)$ is observed with the additive noise

$$
y(n)=x(n)+v(n) .
$$

The following conditions are assumed to hold as.

1) The reflection coefficient series $r(n)$ is unobservable, and is zero-mean, independent and identically distributed (IID), and a non-Gaussian process with at least one cumulant $0<\left|\gamma_{k w}\right|<\infty(k>2)$, where $\gamma_{k w}$ denotes the $k$-th order cumulant of the $r(n)$.

2) The additive noise $v(n)$ is a colored Gaussian process independent of input $r(n)$, and hence independent of $x(n)$.

3) The system is causal and of nonminimum phase. The transfer function of the model is assumed to be free of pole-zero cancellations, and is given by

$$
H(z)=\sum_{i=0}^{\infty} h(i) z^{-i}=\frac{\sum_{j=0}^{q} b(j) z^{-j}}{\sum_{i=0}^{q} a(i) z^{-i}},
$$

where $a(p) \neq 0, b(q) \neq 0$ and, $a(0)=b(0)=1$.

Then the seismogram is considered as the output of the ARMA system excited by the reflection coefficient and is degraded by the additive noise. We can achieve the seismic wavelet as long as we get the model parameters. The model order determination plays a significant role.

Our problem is to determine the orders $p$ and $q$ using the fourth-order sample cumulant, and obtain the model parameters $a(i)$ and $b(j)$.

\section{Order determination and parameter estimation}

In system identification where a given sequence represents the output of an ARMA process, the estimations of the proper ARMA model order and parameters are two key problems.

Many techniques have been developed to obtain the model parameters, but most of them assume prior knowledge of the model order. Obviously, it is the first step to select the model order toward the goal of estimating the model parameters. Various order determination methods have been proposed by researches such as Akaike (1974), Cadzow (1982), Giannakis and Mendel (1990), Zhang and Zhang (1993), etc. But the problem of selecting the proper ARMA model order has never been solved satisfactorily.

\subsection{AR order determination}

It is widely recognized that singular value decomposition (SVD) is a numerically robust tool for AR order determination, and works rather well with moderate noise-to-signal ratios (NSRs). The SVD methods for AR order determination of general ARMA models have been proposed by Cadzow (1982), who used autocorrelation for Gaussian processes, and by Giannakis and Mendel (1990), who used cumulants alone for non-Gaussian processes.

The cumulant based method is sensitive to special 
sections which is difficult to choose. In this paper, the author uses the autocorrelation-based method. The autocorrelation is stable, and can be used in MA order determination.

The appropriate matrix is defined as:

$$
\begin{gathered}
\boldsymbol{R}_{\mathrm{e}}= \\
{\left[\begin{array}{cccc}
R\left(q_{\mathrm{e}}+1\right) & R\left(q_{\mathrm{e}}\right) & \cdots & R\left(q_{\mathrm{e}}+1-p_{\mathrm{e}}\right) \\
R\left(q_{\mathrm{e}}+2\right) & R\left(q_{\mathrm{e}}+1\right) & \cdots & R\left(q_{\mathrm{e}}+2-p_{\mathrm{e}}\right) \\
\vdots & \vdots & \vdots & \vdots \\
R\left(q_{\mathrm{e}}+M\right) & R\left(q_{\mathrm{e}}+M-1\right) & \cdots & R\left(q_{\mathrm{e}}+M-p_{\mathrm{e}}\right)
\end{array}\right] .}
\end{gathered}
$$

Theoretical analysis shows that when $M \geq p_{\mathrm{e}}, p_{\mathrm{e}} \geq p$; $q_{\mathrm{e}} \geq q$ and $q_{\mathrm{e}}-p_{\mathrm{e}} \geq q-p$, the rank of $\boldsymbol{R}_{\mathrm{e}}$ is equal to AR order $p$. AR order determination is converted to estimating the effective rank of matrix $\boldsymbol{R}_{\mathrm{e}}$.

In practice, the element $R(m)$ of $\boldsymbol{R}_{\mathrm{e}}$ is replaced by the higher order cumulant estimation $\hat{R}(m)$ of the sample function. As the actual data are limited, the singular values of the matrix $\boldsymbol{R}_{\mathrm{e}}$ will be nonzero. A subjective rule to select the "effective" AR order $p$ is to find the largest drop among the singular values of $\boldsymbol{R}_{\mathrm{e}}$.

\subsection{AR parameters estimation}

AR parameters are estimated by the high-order cumulant based SVD-TLS method. In section 4.1 above, the right singular matrix $\boldsymbol{V}$ and the effective order $p$ of matrix $\boldsymbol{R}_{\mathrm{e}}$ have been obtained. We then construct $(p+1) \times(p+1)$ matrix $\boldsymbol{S}^{(p)}$ (Zhang, 2002) as

$$
\boldsymbol{S}^{(p)}=\sum_{j=1}^{p} \sum_{i=1}^{n+1-p} \sigma_{j j}^{2} \boldsymbol{v}_{j}^{i}\left(\boldsymbol{v}_{j}^{i}\right)^{\mathrm{H}},
$$

where $\boldsymbol{v}_{j}^{k}=[v(k, j), v(k+1, j), \cdots, v(k+p, j)]^{\mathrm{T}}$, and $\boldsymbol{v}(i, j)$ is the element of $\boldsymbol{V}$ in the $i$-th line and the $j$-th column.

The parameters of the AR model are estimated as follows:

$$
a(i)=\frac{\boldsymbol{S}^{(-p)}(i+1,1)}{\boldsymbol{S}^{(-p)}(1,1)},
$$

where $\boldsymbol{S}^{(-p)}$ is the inverse matrix of $\boldsymbol{S}^{(p)}$.

\subsection{MA order determination}

According to AR order determination method, Zhang and Zhang (1993) converted MA order determination to solving the rank of matrix $\boldsymbol{F}_{\mathrm{e}}$. Matrix $\boldsymbol{F}_{\mathrm{e}}$ associated with an extended order $q_{\mathrm{e}}>q$ is constructed as

$$
\boldsymbol{F}_{\mathrm{e}}=\left[\begin{array}{cccc}
f_{k}(0) & f_{k}(1) & \ldots & f_{k}\left(q_{\mathrm{e}}\right) \\
f_{k}(1) & \ldots & f_{k}\left(q_{\mathrm{e}}\right) & 0 \\
\vdots & . & 0 & \vdots \\
f_{k}\left(q_{\mathrm{e}}\right) & 0 & \ldots & 0
\end{array}\right]
$$

where

$$
f_{k}(\tau)=\sum_{i=0}^{p} a(i) C_{k y}(\tau-i, 0, \cdots, 0) .
$$

Theory proved that the $\operatorname{rank}\left(\boldsymbol{F}_{\mathrm{e}}\right)=q+1$. In practice, the elements of $\boldsymbol{F}_{\mathrm{e}}$ are replaced by the estimation $f_{k}(\tau)$. Zhang and Zhang (1993) suggested combining the SVD method and the PODE test to determine the MA order. Simulations demonstrate that the estimated MA order is still not satisfied. The author introduces the information theoretic criteria into MA order determination.

The information theoretic criteria method defines different criteria functions sing information contained in time-series. On the base of the result of MA order determination, we just need a small integer disturbance. Take the parameters $p$ and $q$ as ARMA model orders, when the criteria function is smallest.

The final prediction error (FPE) criteria (Akaike, 1974) function is defined as

$$
F(p, q)=\hat{\sigma}_{\omega p}^{2}\left(\frac{N+p+q+1}{N-p-q-1}\right)
$$

where, $N$ is the length of data, $\hat{\sigma}_{\omega p}^{2}$ is the linear prediction error variance defined as

$$
\hat{\sigma}_{\omega p}^{2}=\sum_{i=0}^{p} \hat{a}_{i} \hat{R}_{x}(q-i) .
$$

\subsection{MA parameters estimation}

There have been various methods used to estimate MA parameters, such as the R-C (autocorrelationcumulant) algorithm, W-slice algorithm and $C(q, n)$ algorithm. In order to suppress the additive noise completely, the author uses a cumulant-based method in this section. The method has two forms as follows:

$$
\begin{gathered}
\sum_{i=0}^{q} b(i) c_{k y}^{3}(q, i-\tau)=c_{k y}(\tau, 0) c_{k y}(q, 0) c_{k y}(q, q), \\
\sum_{i=0}^{q} b^{3}(i) c_{k y}(q, i-\tau)=c_{k y}(\tau, 0) \frac{c_{k y}(q, q)}{c_{k y}(q, 0)} .
\end{gathered}
$$

In the actual data processing, it is necessary to run these two forms at the same time. If the value $b(i)$ in equation (11) is much smaller than one, the first form is a failure. Similarly, the second form fails when $b^{3}(i)$ is far smaller than one in equation (12).

MA parameters $b(i)(i=1,2, \cdots, q)$ are estimated via solving $b(i)$ and $b^{3}(i)$, obtained separately from equations (11) and (12). 


\section{Numerical simulations and real seis- mic data example}

In this section, the author uses synthetic seismic traces that have various lengths or noises to demonstrate the feasibility of the wavelet extraction approach. For each test case 20 Monte Carlo runs were performed using MATLAB.

The synthetic seismic trace is generated via the causal, non-minimum phase ARMA model filtering the reflection coefficient series as in equation (2), where the probability density function of the reflection coefficient series is modeled as the Bernoulli-Gaussian distribution. The ARMA model's difference equation is

$$
\begin{gathered}
x(t)-1.6 x(t-1)+0.8 x(t-2)= \\
r(t)-0.8 r(t-1)-1.2 r(t-2) .
\end{gathered}
$$

\subsection{Order determination}

The approach proposed in this paper is applied in the post-stack data. Most noise is reduced by superposition. In this simulation, colored Gaussian additive noise whose noise-to-signal ratios are $10 \%$ and $30 \%$ are added to the seismic trace with the length of $2 \mathrm{~s}$ and $10 \mathrm{~s}(1 \mathrm{~ms}$ sample). The correct times of model orders obtained from these data sets are displayed in Table 1.

\begin{tabular}{|c|c|c|c|c|c|c|}
\hline \multirow{3}{*}{$\begin{array}{l}\text { Length } \\
\quad N\end{array}$} & \multirow{2}{*}{\multicolumn{2}{|c|}{$\begin{array}{c}\text { AR order } \\
\text { determination for } \\
\text { different NSRs }\end{array}$}} & \multicolumn{4}{|c|}{ MA order determination for different NSRs } \\
\hline & & & \multicolumn{2}{|c|}{ Cumulant-based method } & \multicolumn{2}{|c|}{ The new approach } \\
\hline & $10 \%$ & $30 \%$ & $10 \%$ & $30 \%$ & $10 \%$ & $30 \%$ \\
\hline 2000 & 20 & 20 & 16 & 16 & 20 & 20 \\
\hline 10000 & 20 & 20 & 19 & 19 & 20 & 20 \\
\hline
\end{tabular}

Table 1 Order determination

As Table 1 shows, AR order determination can obtain satisfied results with different lengths and moderate noises. However the cumulant-based method is sensitive to the length of data, the result is not satisfied even combining the SVD method and the PODE (product of diagonal elements) test. The new approach proposed in this paper can improve the precision of the cumulant-based method greatly.

\subsection{Sensitivity to the noise of parameters estimation}

After getting the correct model orders, we consider the factors in estimating the model parameters. In this situation, colored Gaussian noises with various NSRs $(10 \%$ and $30 \%)$ are considered to test the sensitivity to the noise of parameters estimation. The synthetic seismic traces with various noises are shown in Figure 1.
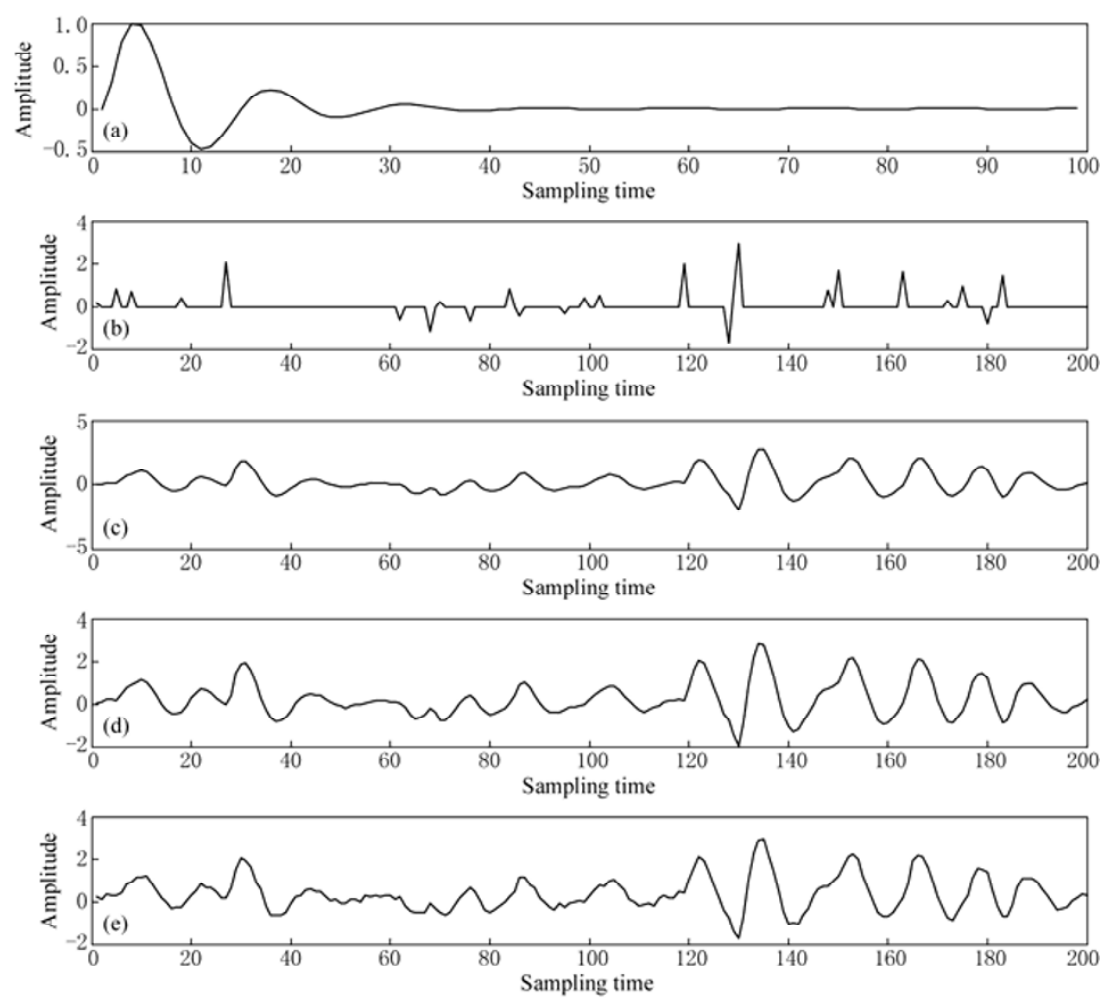

Figure 1 Sections of synthetic seismic traces with various noises. (a) The original wavelet; (b) The reflection coefficient series; (c) The synthetic trace (noise free); (d) The synthetic trace with NSR of $10 \%$; (e) The synthetic trace with NSR of $30 \%$. 
The parameters of wavelets estimated from traces in Figures 1d and 1e are shown in Table 2.

As Table 2 shows, the estimated parameters approach to the true values with small variance. The extracted waveforms are consistent with those shown in Figure 2.

Table 2 Parameters estimation (various SNRs)

\begin{tabular}{lrrrrrr}
\hline \multirow{2}{*}{$\begin{array}{l}\text { Parameters and } \\
\text { their true value }\end{array}$} & \multicolumn{2}{c}{$\operatorname{NSR}\left(\sigma_{\mathrm{n}}^{2} / \sigma_{\mathrm{s}}^{2}=10 \%\right)$} & & \multicolumn{2}{c}{$\operatorname{NSR}\left(\sigma_{\mathrm{n}}^{2} / \sigma_{\mathrm{s}}^{2}=30 \%\right)$} \\
\cline { 2 - 4 } \cline { 5 - 6 } & & \multicolumn{1}{c}{$E\{\cdot\}$} & $\sigma_{2} /\left(10^{-3}\right)$ & & \multicolumn{1}{c}{$E\{\}$} & $\sigma_{2} /\left(10^{-3}\right)$ \\
\hline$a(1)$ & -1.6 & -1.6018 & 0.04765 & & -1.5988 & 0.07452 \\
$a(2)$ & 0.8 & 0.8006 & 0.05223 & & 0.7978 & 0.05122 \\
$b(1)$ & -0.8 & -0.8091 & 3.2 & & -0.8314 & 13.7 \\
$b(2)$ & -1.2 & -1.1889 & 26.3 & & -1.1740 & 126 \\
\hline
\end{tabular}

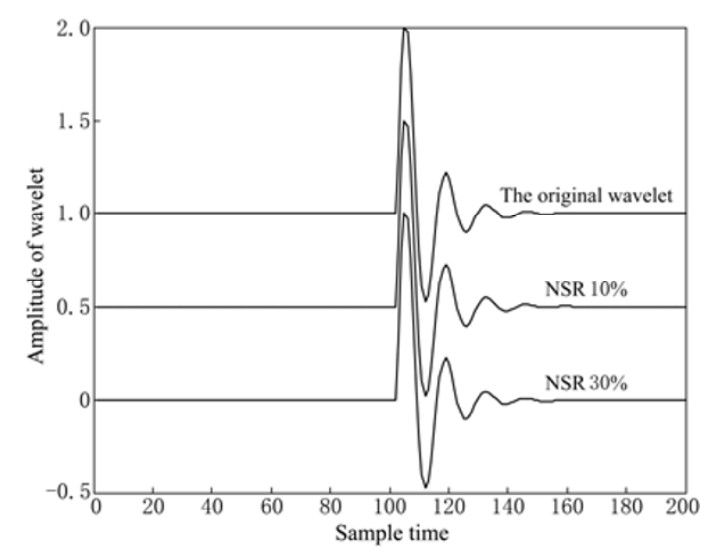

Figure 2 Wavelets estimated from seismic traces contaminated by colored non-Gaussian noise with different power ratio.

\subsection{Sensitivity to the length of parameters estimation}

In this simulation, the observed data series with their length of 2000 and 10000 are considered to test the sensitivity to the length of model parameters estimation.

Table 3 Parameter estimation (various lengths $N$ )

\begin{tabular}{lrrrrrr}
\hline \multirow{2}{*}{$\begin{array}{l}\text { Parameters and } \\
\text { their true value }\end{array}$} & \multicolumn{2}{c}{$N=2000$} & \multicolumn{2}{c}{$N=10000$} \\
\cline { 2 - 4 } \cline { 5 - 6 } & & \multicolumn{1}{c}{$E\{\cdot\}$} & $\sigma^{2} /\left(10^{-3}\right)$ & & $E\{\cdot\}$ & $\sigma^{2} /\left(10^{-3}\right)$ \\
\hline$a(1)$ & -1.6 & -1.5998 & 0.3348 & & -1.5977 & 0.06185 \\
$a(2)$ & 0.8 & 0.7975 & 0.3183 & & 0.7978 & 0.06888 \\
$b(1)$ & -0.8 & -0.8715 & 18.2 & & -0.8147 & 12.2 \\
$b(2)$ & -1.2 & -1.1705 & 20.3 & -1.2053 & 11.1 \\
\hline
\end{tabular}

As Table 3 shows, the estimated AR parameters approach to the true values. However the MA parameters are not satisfied when the length of data is short. The waveforms extracted from different traces with various lengths are consistent. This demonstrates the effectiveness of the method proposed in this paper. The waveforms extracted from different length shown in Figure 3 are continuous.

\subsection{Real seismic data examples}

In this section, real field data as shown in Figure 4 is used to test the feasibility of this approach. In Figure 1 , the seismic section consists of 101 traces, from 0 to 2 $\mathrm{s}$, and the sampling time is $1 \mathrm{~ms}$.

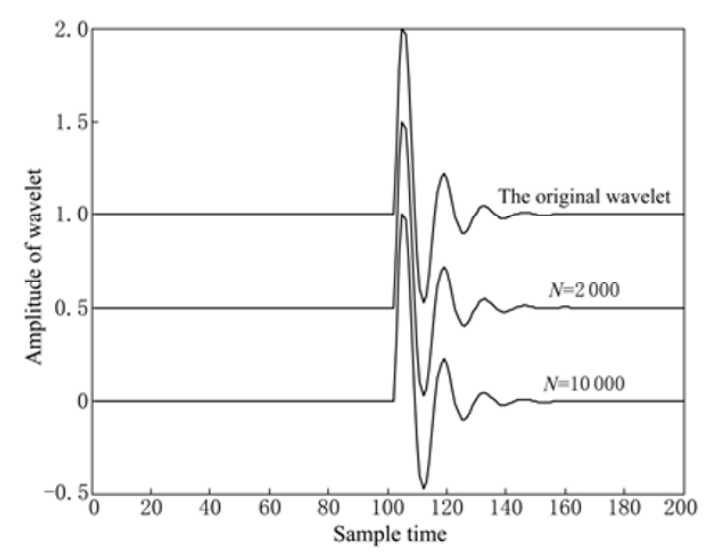

Figure 3 Wavelets estimated from seismic traces with different lengths $(N)$.

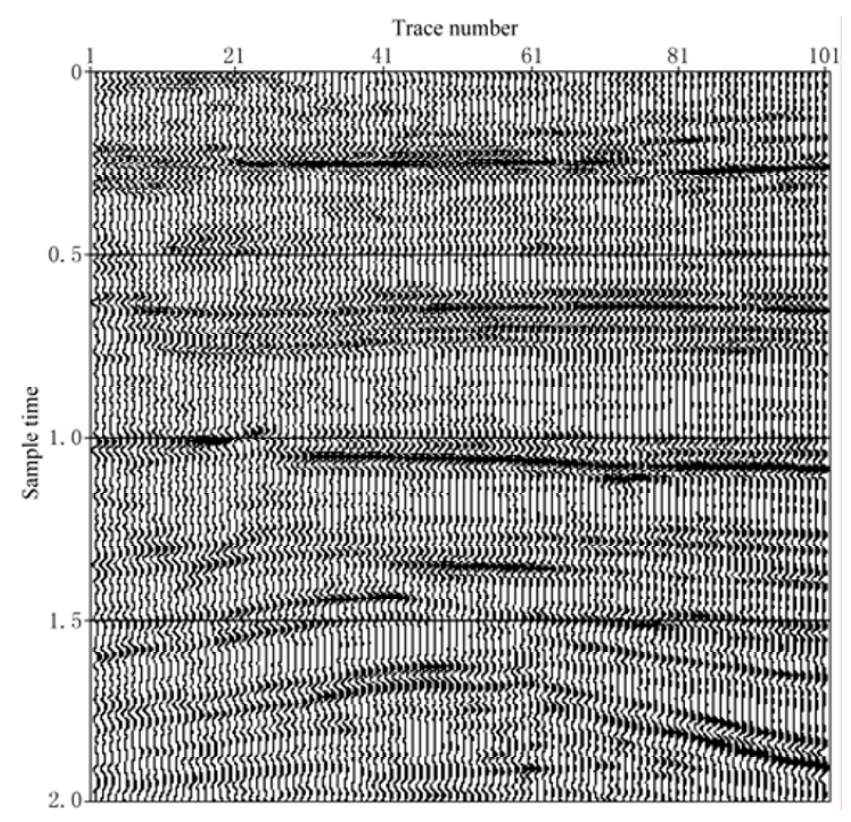

Figure 4 The real seismic section.

Ten adjacent traces of data are selected randomly as a set, and then the wavelet is obtained as an average. As Figure 5 shows, the waveforms extracted from different seismic traces sections are very consistent. This proves the stability of the extraction approach proposed in this paper. 


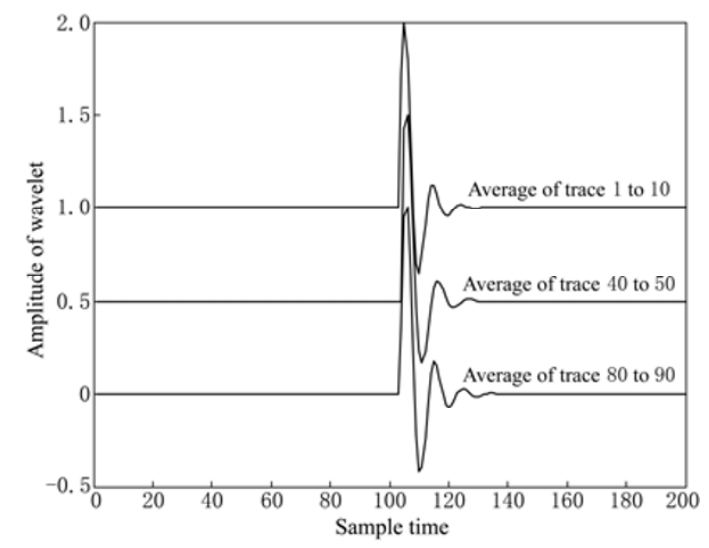

Figure 5 Wavelet estimation from traces in Figure 3.

\section{Conclusions}

According to the results of theoretical analysis and the numerical simulations of this method, we can draw some conclusions as follows.

1) The autocorrelation-based ARMA model seismic wavelet method could provide an economical, fast and consistent signal modeling in fitting seismic traces.

2) The cumulant-based method estimates the MA order badly even when combining the SVD method and the PODE test. The new approach proposed in this paper can improve the precision of cumulant-based method. The new approach just introduces the PEF method into the cumulant-based method in order to determine MA order. We can try introducing some other information theoretic criteria methods.

3) Because of the length of data and the additive noise, the MA order and parameters obtained are not very good. Non-linear optimization can be used to get a high resolution wavelet only through adjusting MA parameters.

Acknowledgements This work was supported by the National High Technology Research and Development Program of China (863 Program, No. 2007AA09Z301), the Graduate Innovation Fund of China University of Petroleum and National Natural Science Foundation of China (40974072).

\section{References}

Akaike H (1974). A new look at the statistical model identification. IEEE Trans Automatic Control 19: 716-723.

Cadzow J A (1982). Spectral estimation: An overdetermined rational model equation approach. IEEE 70(9): 907-939.

Dai Y S, Zheng D L, Wei L and Huo Z Y (2006). Estimation of high-order statistic seismic wavelet. Oil Geophysical Prospection 41(5): 514-518.

Giannakis G B and Mendel J M (1990). Cumulant-based order determination of non-Gaussian ARMA models. IEEE Transactions on Signal Processing 38: $1411-1423$

Lazear G D (1993). Mixed-phase wavelet estimation using fourth-order cumulants. Geophysics 58(7): 1042-1 051.

Velis D R and Ulrych T J (1996). Simulated annealing wavelet estimation via fourth-order cumulant matching. Geophysics 61(6): 1939-1 948.

Zhang X D (2002). Modern Signal Processing. Tsinghua University Publishing House, Beijing, 80-96 (in Chinese).

Zhang X D and Zhang Y S (1993). Singular value decomposition-based MA order determination of non-Gaussian ARMA model. IEEE Transactions on Signal Processing 41(8): 2 657-2 664. 